\title{
DAS User Manual
}

\author{
Roy Østensen \\ Instituut voor Sterrenkunde, K.U. Leuven \\ B-3001 Leuven, Belgium \\ e-mail: roy@ster.kuleuven.be \\ http://newton.ster.kuleuven.be/ roy/helas/
}





\section{HELAS Database for AsteroSeismology}

\subsection{Introduction}

The HELAS Database for AsteroSeismology (DAS) is one of the deliverables of the Work Package NA5: Asteroseismology of the European Coordination Action in Helio and Asteroseismology $\left(\mathrm{HELAS}^{1}\right)$. The DAS aims to provide easy access to publicly available asteroseismological timeseries data, both photometric and spectroscopic. In particular, the DAS and the HELAS software package FAMIAS are ideally suited to train Master and PhD students in asteroseismic data analysis and to build longterm datasets. The number of stars in the system is still limited and reflects the willingness of data owners to provide their data after publication. Work continues to populate the database with contributions from the community, and at present the number of stars in the database is 82 (Tables 1 and 2).

\subsection{Features}

Before getting access to the database, the user must agree to the conditions of use, which obliges the user to refer to a source publication provided with each dataset, whenever archive data is used in an article. The database search interface (Figure 1) includes search by variable class, name or coordinates. Output tables (Figure 2) are generated in HTML with links to automatically generated finding charts, the Aladin viewer, and a detailed data sheet (Figure 3) that displays catalogue data for each target, together with a DSS image of the source. All stars have been added with a number of identifiers including the common or constellation name, HD catalog number, Hipparcos number, BD catalog name and others, making it easy to find a particular star in the database. The database currently recognises 15 different classes of variable stars, but not all classes have any entries yet. Table 3 provides the keys to the variable star classes used in the database (and in Tables 1 and 2), and also summarises the total number of stars in each class.

\footnotetext{
${ }^{1}$ http://www.helas-eu.org
} 


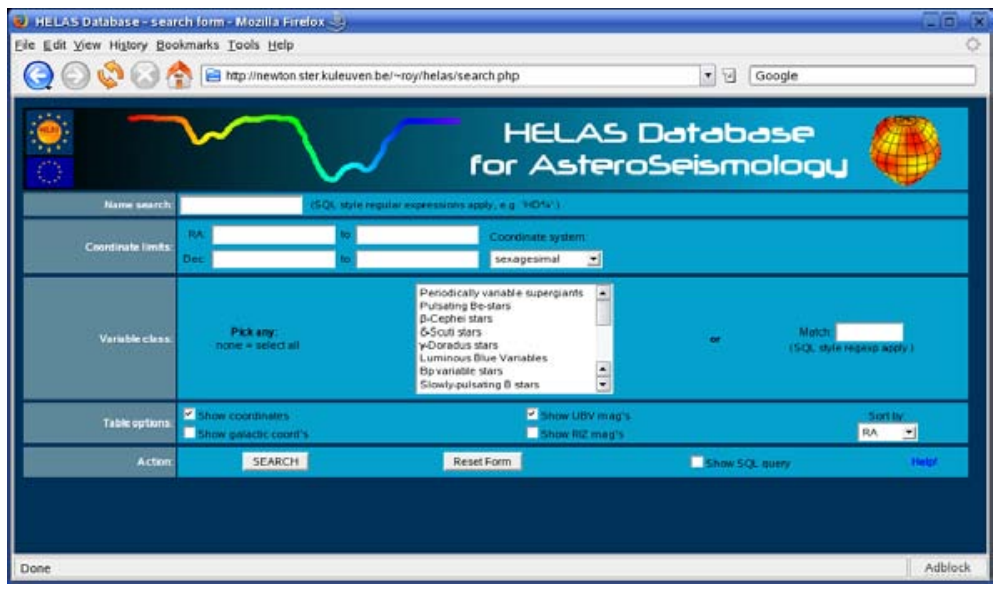

Figure 1: The database search interface.

\subsection{Archive data}

At the bottom of the data sheet, a table is generated with all datasets for this star in the database (see Figure 3 for an example). Each dataset is associated with a type identifier, usually 'spectra' or 'rv' to distinguish between sets that contain actual spectroscopy (usually a small section of a high resolution spectrum or several lines combined into one) or radial velocities derived from spectroscopy. Photometric datasets have also been included, and since they can be very different in nature, they have been given different identifiers such as 'most', 'ultracam', 'geneva', 'wet' and so on. In Table 1, several stars with both spectroscopic and photometric data are listed.

\subsection{Referencing}

With each dataset entry, there is an associated README file, which, in addition to describing the format of the data provided, gives one or more references to articles that use and describe the dataset. This reference, or the most important one if there are several, is also provided in the data table as a link that will lead to the relevant paper. The final entry in the dataset table is a link to the actual dataset, normally a compressed TAR-file. The dataset can contain either tables of timeseries data or in the case of spectra, individual files for each measurement. The README-file is always included in the archive file. 


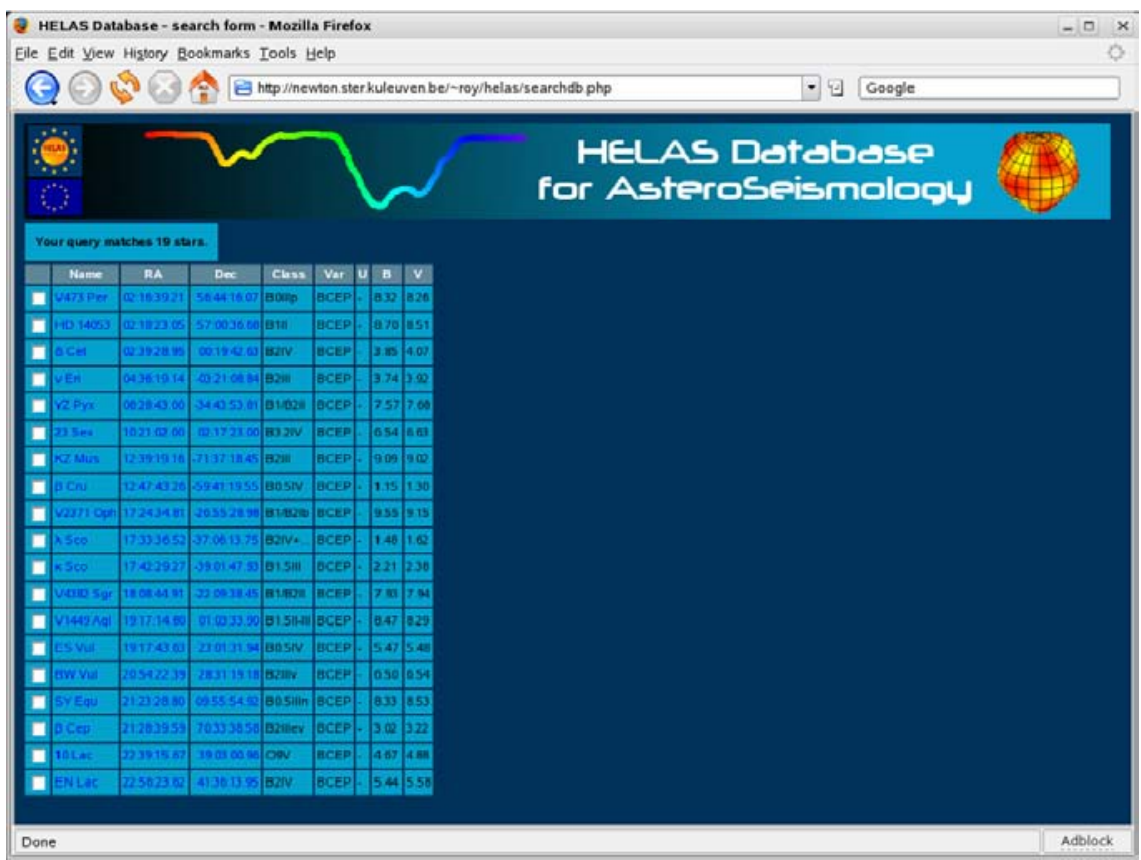

Figure 2: The result of a search for $\beta$ Cephei stars in the database.

\subsection{Platform}

The database runs under MySQL (www.mysql.com; an open source database) with interfaces in Perl for uploading the database entries. The WWW interface uses HTML forms and tables generated by PHP. The DAS is hosted at http://newton.ster.kuleuven.be/ roy/helas/ and also available from the HELAS platform ${ }^{2}$ through the NA5 website link.

\subsection{Call for Contributions}

Anybody who wishes to contribute published data, spectroscopic or photometric, on any particular star, is kindly asked to contact the author by e-mail to roy@ster.kuleuven.be.

\footnotetext{
${ }^{2}$ http://www.helas-eu.org
} 


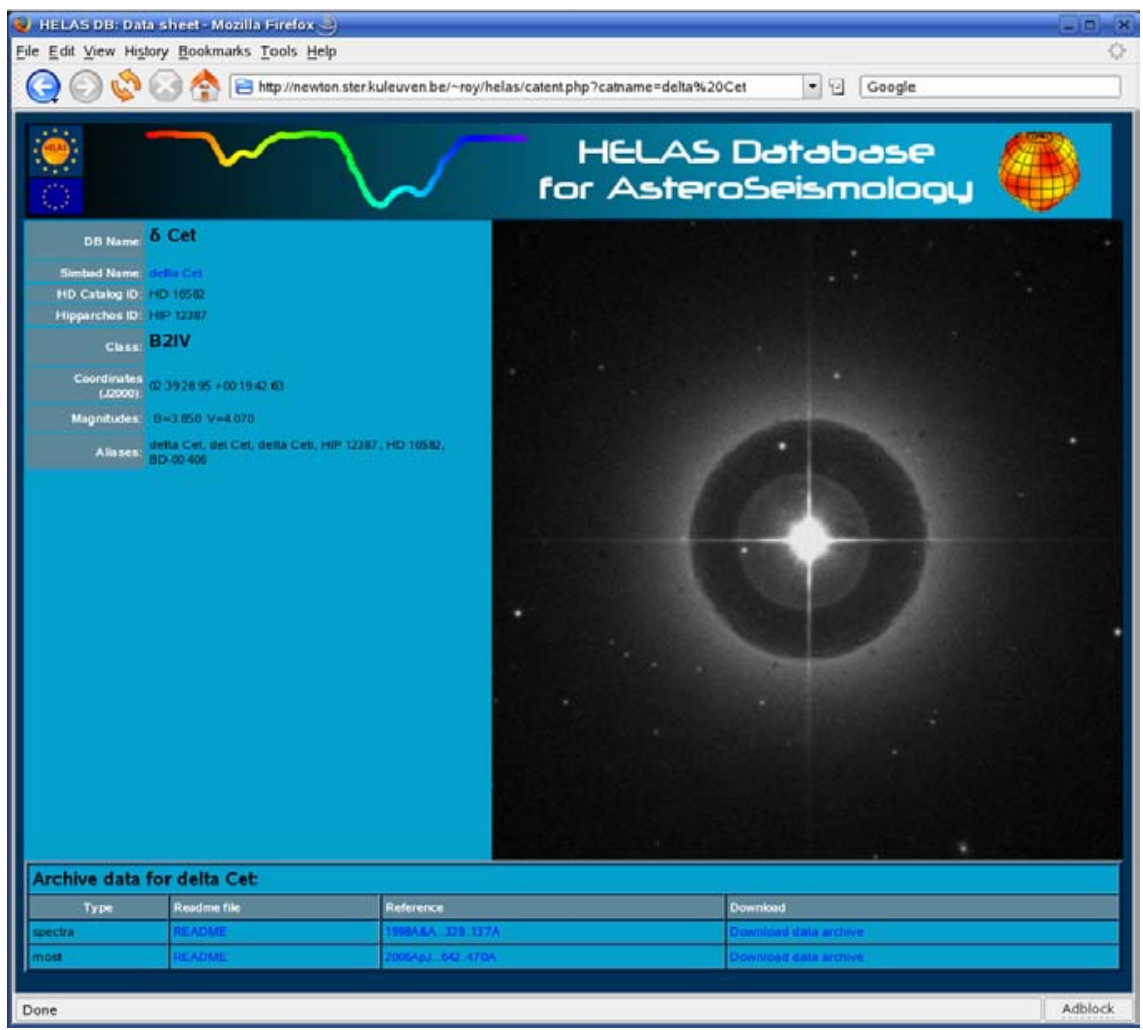

Figure 3: An example data sheet, here for the $\beta$ Cep star $\delta$ Ceti.

\section{Acknowledgements}

$\mathrm{R} \varnothing$ has been supported by the FP6 European Coordination Action HELAS and by the Research Council of the University of Leuven under grant GOA/2003/04. 
Table 1: Variable stars with data in the DAs.

\begin{tabular}{|c|c|c|c|}
\hline DB name & HD number & Class & Data type \\
\hline HD 215 & HD 215 & GDOR & geneva \\
\hline HD 277 & HD 277 & GDOR & geneva \\
\hline EK Psc & & SDBV & ultracam \\
\hline V746 Cas & HD 1976 & SPB & geneva \\
\hline HD 2842 & HD 2842 & GDOR & geneva \\
\hline $53 \mathrm{Psc}$ & HD 3379 & SPB & geneva \\
\hline PG $0101+039$ & & SDBV & most \\
\hline HD 7169 & HD 7169 & GDOR & geneva \\
\hline FO Cet & HD 12901 & GDOR & spectra, geneva \\
\hline V354 Per & HD 13745 & SPB & geneva \\
\hline V473 Per & HD 13831 & BCEP & geneva \\
\hline HD 14053 & HD 14053 & BCEP & geneva \\
\hline delta Cet & HD 16582 & BCEP & spectra, most \\
\hline 53 Ari & HD 19374 & SPB & geneva \\
\hline V576 Per & HD 21071 & SPB & geneva \\
\hline IP Per & HD 278937 & DSCUT & multisite \\
\hline HD 23874 & HD 23874 & GDOR & geneva \\
\hline tau08 Eri & HD 24587 & SPB & spectra \\
\hline DO Eri & HD 24712 & WR & wet \\
\hline V1133 Tau & HD 25558 & SPB & geneva \\
\hline GU Eri & HD 26326 & SPB & spectra \\
\hline V1143 Tau & HD 28114 & SPB & geneva \\
\hline V1144 Tau & HD 28475 & $\mathrm{SPB}$ & geneva \\
\hline nu Eri & HD 29248 & BCEP & spectra \\
\hline V350 CMa & HD 48501 & GDOR & spectra, geneva \\
\hline V450 Car & HD 53921 & SPB & spectra \\
\hline MM CMa & HD 55522 & BPV & spectra \\
\hline DO Lyn & HD 62454 & GDOR & geneva \\
\hline rho Pup & HD 67523 & DSCUT & spectra \\
\hline NO Vel & HD 69144 & SPB & spectra \\
\hline EF Lyn & HD 69715 & GDOR & geneva \\
\hline YZ Pyx & HD 71913 & BCEP & geneva \\
\hline HY Vel & HD 74560 & SPB & spectra \\
\hline omicron Vel & HD 74195 & SPB & spectra \\
\hline HD 74504 & HD 74504 & GDOR & geneva \\
\hline V335 Vel & HD 85953 & SPB & spectra \\
\hline HD 86358 & HD 86358 & GDOR & geneva \\
\hline $23 \mathrm{Sex}$ & HD 89688 & BCEP & geneva \\
\hline V514 Car & HD 92287 & SPB & spectra \\
\hline xi Hya & HD 100407 & SLR & rv \\
\hline V863 Cen & HD 105382 & BPV & spectra \\
\hline
\end{tabular}


Table 2: Variable stars (cont'd.)

\begin{tabular}{|c|c|c|c|}
\hline DB name & HD number & Class & Data type \\
\hline HD 105458 & HD 105458 & GDOR & geneva \\
\hline FG Vir & HD 106384 & DSCUT & spectra \\
\hline DD CVn & HD 108100 & GDOR & geneva \\
\hline KZ Mus & HD 109885 & BCEP & geneva \\
\hline beta Cru & HD 111123 & BCEP & spectra \\
\hline MP Com & HD 113867 & GDOR & geneva \\
\hline V869 Cen & HD 123515 & SPB & spectra \\
\hline alfa Cen B & HD 128621 & SLR & rv \\
\hline V1019 Cen & HD 131120 & BPV & spectra \\
\hline FK Boo & HD 138003 & SPB & geneva \\
\hline IU Lib & HD 138764 & SPB & spectra \\
\hline d Lup & HD 138769 & BPV & spectra \\
\hline PT Ser & HD 140873 & SPB & spectra \\
\hline epsilon Oph & HD 146791 & SLR & rv \\
\hline zeta Oph & HD 149757 & $\mathrm{BE}$ & most \\
\hline $\mathrm{J} 1717+5805$ & & SDBV & ultracam \\
\hline V2371 Oph & HD 157485 & BCEP & geneva \\
\hline lambda Sco & HD 158926 & BCEP & spectra \\
\hline kappa Sco & HD 160578 & BCEP & spectra \\
\hline HD 163830 & HD 163830 & SPB & most \\
\hline HD 163899 & HD 163899 & PVSG & most \\
\hline V3984 Sgr & HD 163868 & $\mathrm{BE}$ & most \\
\hline V4382 Sgr & HD 165812 & BCEP & geneva \\
\hline V1402 Aql & HD 177230 & WR & most \\
\hline V4198 Sgr & HD 177863 & SPB & spectra \\
\hline V338 Sge & HD 169820 & $\mathrm{SPB}$ & geneva \\
\hline V1449 Aql & HD 180642 & BCEP & geneva \\
\hline $2 \mathrm{Vul}$ & HD 179588 & SPB & geneva \\
\hline ES Vul & HD 180968 & BCEP & geneva \\
\hline V4199 Sgr & HD 181558 & SPB & spectra \\
\hline V377 Vul & HD 182255 & SPB & geneva \\
\hline V1473 Aql & HD 191295 & SPB & geneva \\
\hline BW Vul & HD 199140 & BCEP & spectra \\
\hline SY Equ & HD 203664 & BCEP & geneva \\
\hline beta Cep & HD 205021 & BCEP & spectra \\
\hline HD 206540 & HD 206540 & SPB & geneva \\
\hline $16 \mathrm{Peg}$ & HD 208057 & $\mathrm{SPB}$ & geneva \\
\hline $10 \mathrm{Lac}$ & HD 214680 & BCEP & geneva \\
\hline xi Oct & HD 215573 & SPB & spectra \\
\hline EN Lac & HD 216916 & BCEP & spectra \\
\hline V394 And & HD 222555 & SPB & geneva \\
\hline
\end{tabular}


Table 3: Variable star classes used in the DAS.

\begin{tabular}{lll}
\hline Class & Reference & Total \\
\hline PVSG & Periodically variable supergiants & 1 \\
BE & Pulsating Be-stars & 2 \\
BCEP & $\beta$-Cephei stars & 19 \\
DSCUT & $\delta$-Scuti stars & 3 \\
GDOR & $\gamma$-Doradus stars & 14 \\
LBV & Luminous Blue Variables & 0 \\
BPV & Bp variable stars & 4 \\
SPB & Slowly-pulsating B stars & 30 \\
SLR & Solar-like oscillations in red giants & 3 \\
SDBV & Pulsating subdwarf B stars & 3 \\
DAV & Pulsating DA white dwarfs & 0 \\
GWVIR & GW-Virginis stars & 0 \\
ROAP & Rapidly oscillating Ap stars & 0 \\
WR & Wolf-Rayet stars & 2 \\
CV & Cataclysmic variables & 0 \\
\hline
\end{tabular}


